ARTICLE

\title{
Contact-electro-catalysis for the degradation of organic pollutants using pristine dielectric powders
}

\author{
Ziming Wang ${ }^{1,2,6}$, Andy Berbille (1) 1,2,3,6, Yawei Feng ${ }^{1,2}$, Site $\mathrm{Li}^{4}$, Laipan Zhu (1) 1,2, Wei Tang (1) 1,2凶 \& \\ Zhong Lin Wang (1) $1,2,5 \bowtie$
}

Mechanochemistry has been studied for some time, but research on the reactivity of charges exchanged by contact-electrification (CE) during mechanical stimulation remains scarce. Here, we demonstrate that electrons transferred during the CE between pristine dielectric powders and water can be utilized to directly catalyze reactions without the use of conventional catalysts. Specifically, frequent CE at Fluorinated Ethylene Propylene (FEP) - water interface induces electron-exchanges, thus forming reactive oxygen species for the degradation of an aqueous methyl orange solution. Contact-electro-catalysis, by conjunction of $\mathrm{CE}$, mechanochemistry and catalysis, has been proposed as a general mechanism, which has been demonstrated to be effective for various dielectric materials, such as Teflon, Nylon-6,6 and rubber. This original catalytic principle not only expands the range of catalytic materials, but also enables us to envisage catalytic processes through mechano-induced contactelectrification.

\footnotetext{
${ }^{1}$ CAS Center for Excellence in Nanoscience, Beijing Institute of Nanoenergy and Nanosystems, Chinese Academy of Sciences, Beijing 100083, China. ${ }^{2}$ School of Nanoscience and Technology, University of Chinese Academy of Sciences, Beijing 100049, China. ${ }^{3}$ CAS Center for Excellence in Nanoscience, National Center for Nanoscience and Technology (NCNST), Beijing 100190, China. ${ }^{4}$ Department of Chemistry, Carnegie Mellon University, Pittsburgh, PA 15213, USA. ${ }^{5}$ School of Materials Science and Engineering, Georgia Institute of Technology, Atlanta, GA 30332-0245, USA. ${ }^{6}$ These authors contributed equally: Ziming

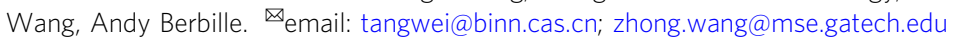


$\mathrm{M}$ echanochemistry was regarded as one of the ten changing world technologies by IUPAC, ${ }^{1}$ which generally relies on the increase of defects, ${ }^{2-4}$ local extreme conditions, ${ }^{5-7}$ or force-induced effects ${ }^{8-10}$ under external mechanical agitations to facilitate reactions. However, little attention has been paid to the potential contribution made by the contact electrification effect between liquid and solid, despite the frequent contacts and separations occurring during mechanochemical processes. Previous researches have demonstrated that organic pollutants can be degraded by magnetic stirring with piezoelectric materials, which is referred to as tribocatalysis. ${ }^{1-13}$ However, the utilization of piezoelectric materials in these studies actually makes the interpretation of the underlying mechanism ambiguous because of the presence of both piezoelectric and triboelectric effects. For example, the degradation mechanism by the same method has also been described as hydromechanics-induced piezocatalysis (Supplementary Table 1). ${ }^{14-16}$ To unambiguously understand the mechanism for general materials, solid evidence regarding the contributions made specifically by contact electrification to the chemical reactions are required. Pristine polymers represent an ideal choice for this purpose. Unlike conventional catalysts, such as metals, ${ }^{17,18}$ zeolites, ${ }^{19,20}$ semiconductors, ${ }^{21,22}$ and piezoelectric materials, ${ }^{23,24}$ pristine polymers, due to their stable electronic structures, have rarely been considered as suitable materials for catalysis (Supplementary Table 2). Recently, it has been demonstrated that dielectric materials can withdraw electrons from DI water during the contact electrification process, and intensive efforts have been directed towards the mechanism and application of liquid-solid CE in the meantime. ${ }^{25-28}$ Electrons were proved to contribute and in a majority of cases dominate the charge transfer process during $\mathrm{CE}$ at the waterdielectric interface, ${ }^{29}$ which implies that promoting reaction rate by CE should be possible.

Here, we first demonstrated that electrons exchanged during $\mathrm{CE}$ at the interface of water and dielectric powder could be utilized in chemical reactions, through a process called contactelectro-catalysis (CEC). The mechanism of CEC proposes that frequent contact-separation cycles at the surface of dielectric powder are induced by the growth and collapse of cavitation bubbles during mechano-stimulation, ${ }^{30}$ and electrons exchanged during such CE process could be transferred to different substrates to form reactive oxygen species (ROS). These CEC-yielded ROS in an aqueous solution can then react with refractory organic compounds in advanced oxidation processes (AOPs). The present study mainly focused on the degradation of a 5-ppm aqueous methyl orange (MO) solution in presence of $20 \mathrm{mg}$ of Fluorinated ethylene propylene (FEP) powder. Liquidchromatography mass-spectroscopy (LC-MS) results revealed that $\mathrm{MO}$ was completely degraded after $180 \mathrm{~min}$ of ultrasonication $(40 \mathrm{kHz}, 120 \mathrm{~W})$. Ex-situ morphological and spectroscopic characterizations confirmed that both the physical and chemical properties of FEP powder remained unchanged after degradation. Besides, the electron paramagnetic resonance (EPR) verified the evolution of hydroxyl and superoxide radicals. DFT simulations have also been conducted to evaluate the energy barrier of electron exchange between FEP and water $/ \mathrm{O}_{2}$ in such conditions. Contact-electro-catalysis (CEC), the catalysis of chemical reactions by $\mathrm{CE}$ driven electron exchange, standing at the frontier of mechanochemistry, $\mathrm{CE}$, and catalysis, represents an innovative strategy for yielding ROS and dealing with refractory organic pollutants. A novel wastewater treatment system has been proposed on the basis of CEC principle due to its merits of scalability and recyclability, and we expect more promising reactive systems involving radical species could be established in the future, opening a new field for catalysis.

\section{Results}

Investigations on the degradation of methyl orange. Figure 1 presents the systematic investigation conducted on the degradation of MO. Our experiment design is illustrated in Fig. 1a. $20 \mathrm{mg}$ of FEP powder were added to a $50 \mathrm{~mL}$ aqueous solution of $\mathrm{MO}(5$ $\mathrm{ppm}$ ), and then stirred for $48 \mathrm{~h}$ to improve the contact between FEP and water. Thereafter, the as-prepared suspension was ultrasonicated at a frequency of $40 \mathrm{kHz}$ and a power of $120 \mathrm{~W}$. The original light-yellow solution becomes transparent after $3 \mathrm{~h}$, as shown by inserted photos and Supplementary Movie 1. Ultrasonication is employed here for generating cavitation bubbles capable of inducing contact-separation cycles. The existence of contact electrification at the water-FEP interface is supported by the electrical output of a single electrode triboelectric nanogenerator (SE-TENG) that is repeatedly immersed in DI water (Fig. 1b). The magnitude of transferred charges for one single contact-separation cycle between water and FEP film increased from $8.05 \mathrm{nC}$ to saturation at $10.47 \mathrm{nC}$, suggesting charges are induced and accumulated on the FEP surface during contact with DI water. The configuration of the entire setup is depicted in Supplementary Fig. 1. To investigate the discoloration process, aliquots $(2 \mathrm{~mL})$ were sampled at specific intervals and analyzed by $\mathrm{UV}$-Vis spectroscopy. Corresponding optical photos and UV-Vis results are exhibited in Fig. 1c, d respectively. The characteristic absorbance peak of MO decreased as the ultrasonication time increased, and approached zero after $120 \mathrm{~min}$. A control experiment has been conducted under the same condition except for the absence of FEP powder. However, no apparent diminution of absorbance was observed in this case, suggesting that the presence of FEP powder is a prerequisite to initiate the degradation process, see Fig. 1e. Afterward, liquid-chromatography mass-spectroscopy (LC-MS) was employed to identify the degradation products. Figure if depicts the chromatograms of a 5-ppm MO solution at different degradation times, and all peaks are labeled with corresponding mass-to-charge ratios $(\mathrm{m} / \mathrm{z})$. The major peak at a retention time of $8.61 \mathrm{~min}$, with an $\mathrm{m} / z$ of 304 , corresponds to MO. Its intensity diminished along with the formation of other peaks, and all peaks disappeared after $180 \mathrm{~min}$. Detailed analysis of the mass spectra (Supplementary Fig. 2) revealed that these peaks correspond to oxidative degradation products of $\mathrm{MO}$, confirming the contribution from chemical reactions to the degradation. Although FEP is inert to a majority of chemicals, the surface charge density of FEP could reach around $50 \mu \mathrm{C} / \mathrm{m}^{2}$ after contact with water, ${ }^{28}$ and these charges are capable of contributing to chemical reactions. ${ }^{31-33}$ Therefore, various dielectric powders exhibiting different $\mathrm{CE}$ abilities, Polytetrafluoroethylene (PTFE), Polyvinylidene fluoride (PVDF), Nylon-66 (N6), and nitrile butadiene rubber (NBR) were employed here to investigate the relationship between degradation rate and $\mathrm{CE}$ properties (Supplementary Fig. 3). Differences in degradation rates are aligned with discrepancies in CE performances for particles that are negatively charged upon contacting water. FEP, exhibiting the highest surface charge density after contacting with water, is the best performer followed by PTFE and PVDF. It is noteworthy that positively charged particles, such as NBR and N6, show an overall lower rate of degradation than negative ones, and they all exhibit apparent coloration after degradation (Supplementary Fig. 4). This coloration is a consequence of adsorbing of MO at the surface, which can be ascribed to electrostatic attractions between the substrate and charged powder. For instance, MO, an anionic species, can be electrostatically adsorbed on the surface of these positively charged powders. This aggregation is unfavorable to degradation since the direct contact between water and these particles is hindered by accumulated MO, which is consistent with the divergence of degradation rate between positively and negatively charged powder. Degradation products of MO by these 

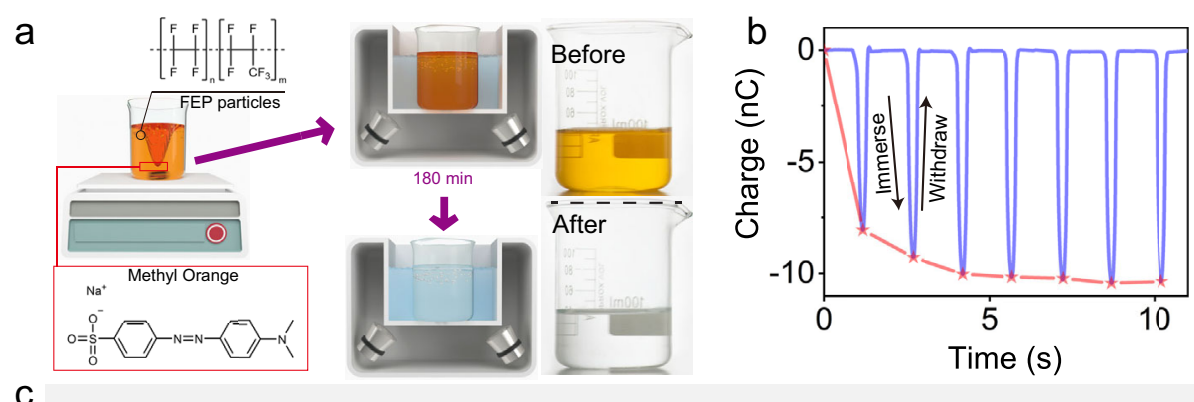

C
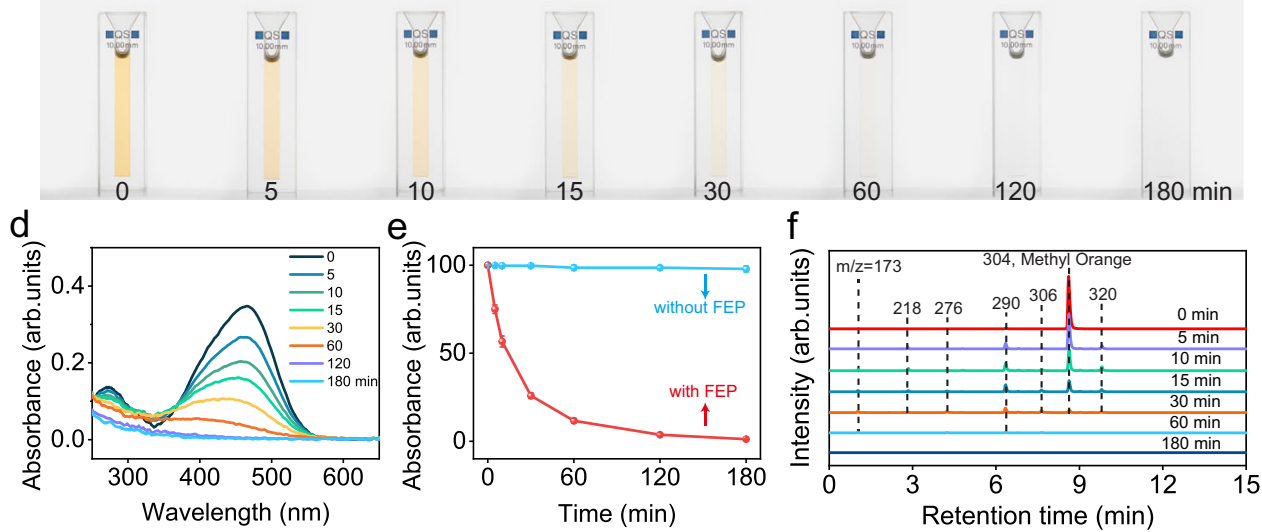

Fig. 1 Degradation of methyl orange by contact-electro-catalysis. a 3D schematic of the experimental setup and protocol. $\mathbf{b}$ Measured electric output of a single electrode TENG that is repeatedly immersed in DI water. c Photographs of MO aqueous solution samples from 0 to $180 \mathrm{~min}$. $\mathbf{d}$ UV-Vis spectra of a $50 \mathrm{~mL}$ aqueous methyl orange (MO) solution during ultrasonication in presence of FEP powder ( $20 \mathrm{mg}$ ) for $3 \mathrm{~h}$. e Comparison of absorbance of $\mathrm{MO}$ solution between situations of with/without FEP powder. $\mathbf{f}$ Mass spectra of the MO solution after separation by liquid-chromatography. Error bars represent standard deviation based on three replicate data.

powders were also analyzed by LC-MS and available in Supplementary Fig. 5. The outperformance of FEP particles for the degradation of $\mathrm{MO}$ inspired us to further explore the essence of CEC.

Characterization of the dielectric powders before/after the reaction. Morphological characterization and element mapping of FEP particles before and after degradation are reported in Fig. 2a, b. Neither obvious coloration nor modifications of the morphology was observed by the naked eye and scanning electron microscopy (SEM). Besides, the inserted mapping pictures indicate that the composition of FEP remained unchanged. A comparison of the particle size distributions before/after the experiment (Fig. 2c) proves that the FEP particles have neither aggregated nor decomposed during the experiment. Apart from morphological characterization, spectroscopic analysis techniques were also performed to deliver more in-depth information on the chemical properties of FEP powder. Figure 2d, e present Raman, and Fourier transform infrared (FTIR) spectroscopy results, respectively. In Raman spectroscopy, the skeleton vibration pattern of FEP before and after the experiment is identical. The fingerprint region in FTIR characterization, below $1500 \mathrm{~cm}^{-1}$, was also stable after the reaction. X-ray photoelectron spectroscopy (XPS) has been conducted to analyze the variation of the chemical state of FEP particles before/after the reaction. The $\mathrm{C} 1 s, \mathrm{~F} 1 s$, and $\mathrm{O} 1 s$ spectra of the FEP powder are listed in Fig. 2f, $\mathrm{h}$, respectively. Neither shift in binding energies of original peaks nor generation of new peaks was observed after degrading MO, which not only further confirms the chemical stability of FEP during CEC, but also excludes the possibility of physical adsorption of MO at FEP surface. These data indicate that the chemically inert FEP powder act as catalysts for the degradation of MO. In addition to the study of MO degradation, investigations on the degradation of Acid Orange17 (AO-17) and Rhodamine $\mathrm{B}(\mathrm{RhB})$ by FEP particles were also proceeded. In the case of AO-17, an anionic dye, the observations were analogous to that of MO (Supplementary Fig. 6). However, the positive RhB ions were adsorbed on the surface of FEP after reaction (Supplementary Fig. 7), which is consistent with the hypothesis that physical adsorption is caused by electrostatic attraction between charged particles and substrates exhibiting reverse polarities, thus corroborating the hypothesis that contact electrification happens during the ultrasonication in presence of FEP.

Generation of the reactive oxygen species (ROS). In order to further understand the underlying mechanism, a series of captures were added separately into the original solution, reaching a final concentration of $1 \mathrm{mM}$. The evolution of $\mathrm{MO}$ concentration in presence of these scavengers is displayed in Fig. 3a. The results indicated that two kinds of radicals, hydroxyl radicals $(\cdot \mathrm{OH})$ and superoxide radicals $\left(\cdot \mathrm{O}_{2}{ }^{-}\right)$, contribute to the degradation of $\mathrm{MO}$. The hydroxyl radical appeared as the limiting factor as only $39.56 \%$ of MO was degraded after $30 \mathrm{~min}$ when it was quenched. The production of reactive radicals was estimated by terephthalic acid (THA) and a water-soluble tetrazolium salt (WST-1) experiment. As depicted in the left panel of Fig. 3b, the emission intensity of THA-OH adduct $(425 \mathrm{~nm})$ increased by 14 -fold over reaction time in presence of FEP powder (Supplementary Fig. 8). Moreover, as depicted in the right panel, the peak of formazan dye $(450 \mathrm{~nm})$ does not appear during the WST test after introducing superoxide dismutase (SOD). (Supplementary Fig. 9). The relationship between the concentration of dissolved $\mathrm{O}_{2}$ and the degradation rate was further explored by constantly bubbling air, $\mathrm{N}_{2}$, or $\mathrm{O}_{2}$ (Fig. 3c). The fastest degradation was achieved while bubbling air (87.6\%), and the lowest when bubbling $\mathrm{N}_{2}(16.5 \%)$. 


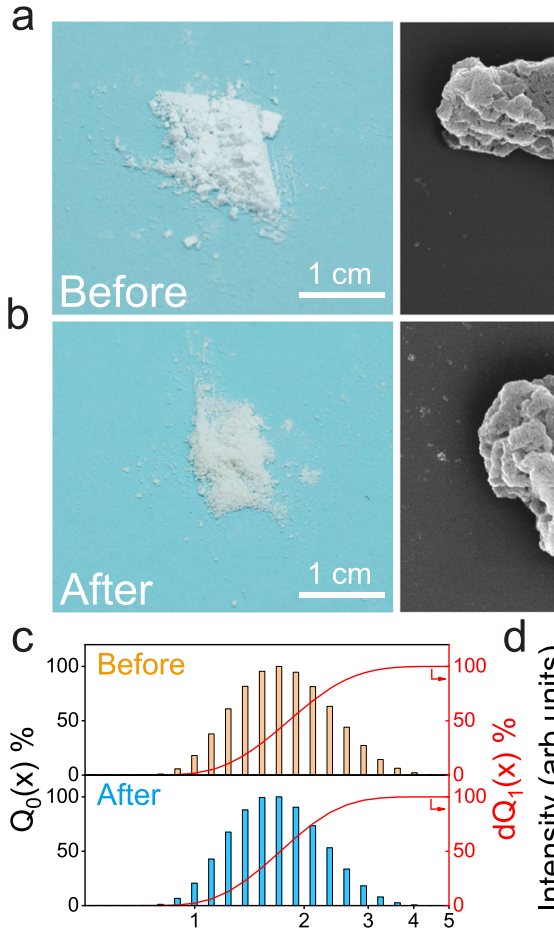

Particle Diameter $(\mu \mathrm{m})$

f

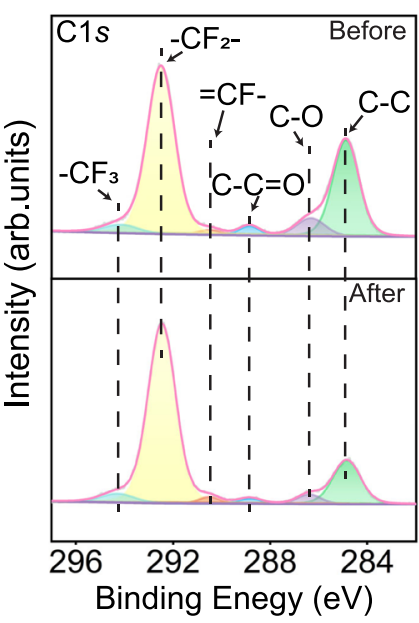

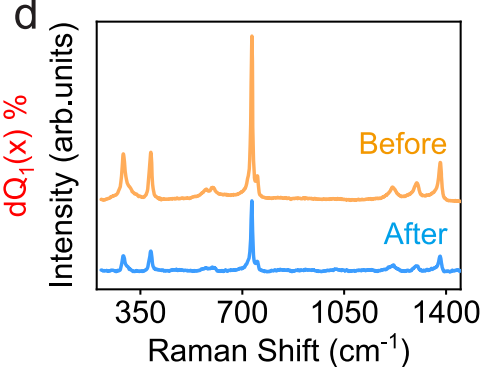

g

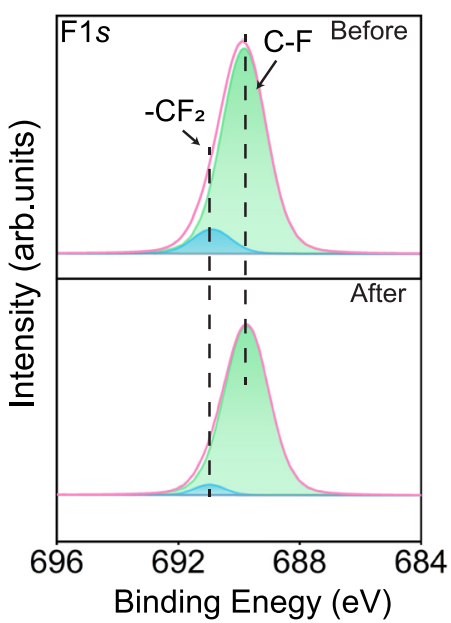

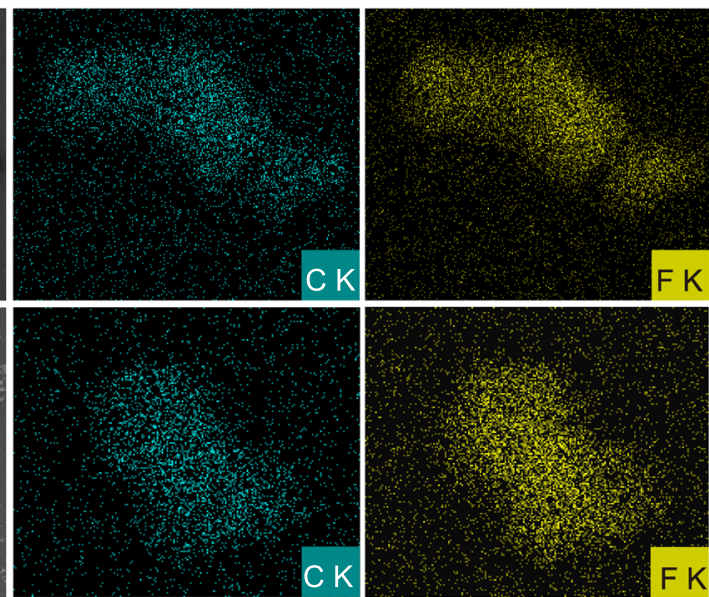

e

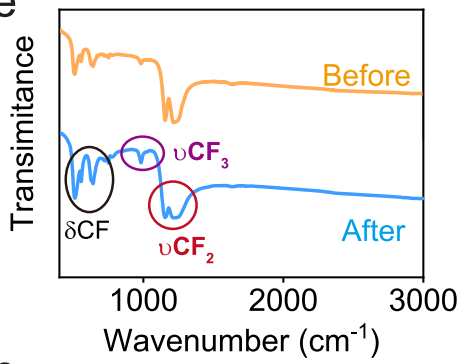

$\mathrm{h}$

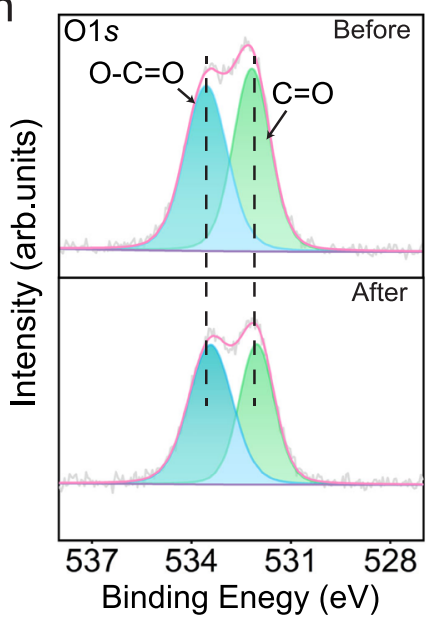

Fig. 2 Characterization of FEP powder before and after contact-electro-catalysis. a Morphological characterization and energy dispersive X-ray (EDX) analysis of the FEP powder before reaction, as well as $\mathbf{b}$, after the reaction. $\mathbf{c}$ Particle size distribution of FEP powder. $\mathbf{d}$ Raman spectra before (orange) and after (blue) the reaction. e Fourier Transform Infrared (FTIR) spectra before (orange) and after (blue) the reaction. $\mathbf{f}$ C1s $\mathbf{g}$ F1s, and $\mathbf{h}$ O1s XPS spectra of FEP powder before and after the reaction.

It is noteworthy that saturating the solution with $\mathrm{O}_{2}$ also hindered the degradation, with the removal of $65.5 \%$. This is mainly attributed to the fact that oxidative atmospheres are detrimental to the contact electrification properties of materials, thus less electrons were induced and transferred during CEC. ${ }^{34}$ Electron paramagnetic resonance spectroscopy (EPR) was also carried out to confirm the production of $\mathrm{O}_{2}{ }^{-}$and $\cdot \mathrm{OH}$ radicals. Two protocols were employed here: a $100 \mathrm{mM}$ DMPO solution, as well as a solution containing both $100 \mathrm{mM}$ DMPO and $1 \mathrm{mM}$ ter-butanol. Ter-butanol was utilized to quench $\cdot \mathrm{OH}$ radicals, enhancing the opportunities for superoxide radicals to react with DMPO, as illustrated by Fig. 3d. No visible peak was measured during ultrasonication without FEP powder, as depicted by the top section of Fig. 3e. In contrast, the curve on the white background infers that quadruplet DMPO-.OH characteristic peaks were yielded when ultrasonication was applied in presence of FEP particles. (Supplementary Fig. 10) And sextuplet DMPO-.OOH peaks were not detected until introducing $1 \mathrm{mM}$ ter-butanol (blue background). The final profile was a superposition of both quadruplet DMPO-.OH peaks and sextuplet DMPO-.OOH peaks, labeled by red stars and orange triangles respectively. The entire profile is dominated by a quadruplet DMPO-.OH peak, which can be ascribed to the fact that hydroxyl radicals are more prone to react with DMPO and that the hydroxyl adduct is more stable than that of superoxide radicals. ${ }^{35}$ Simulated ESR spectra of individual hydroxyl and superoxide radicals, as well as their superposition with respective weights of 70 and $30 \%$, are displayed in Fig. 3f. Raw code for simulation is listed in Supplementary Note 1 . The calculated EPR spectra are consistent with acquired data in Fig. 3e, and divergences between them are 


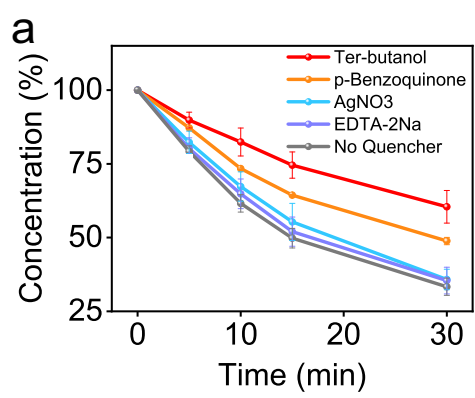

d

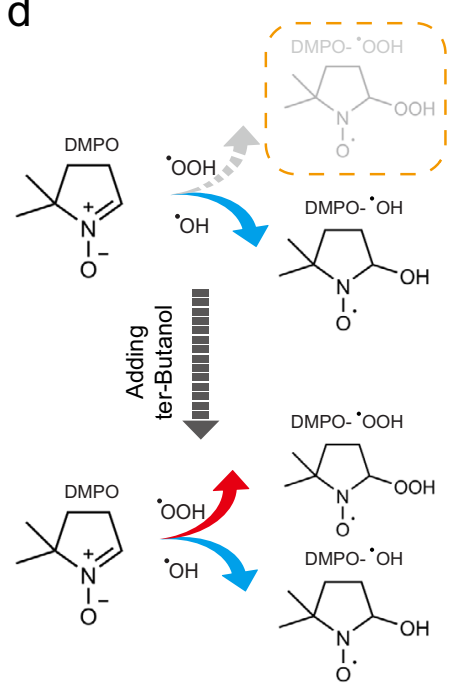

$b$

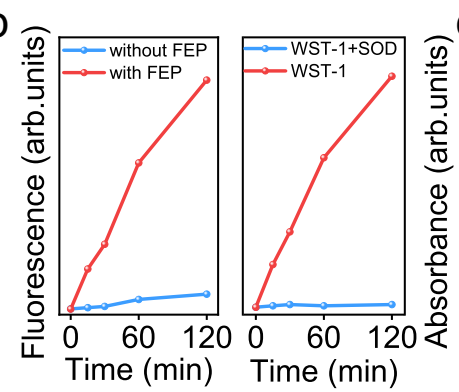

e

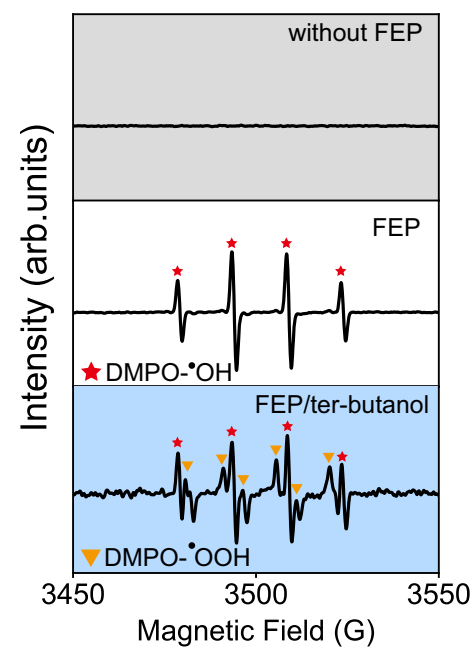

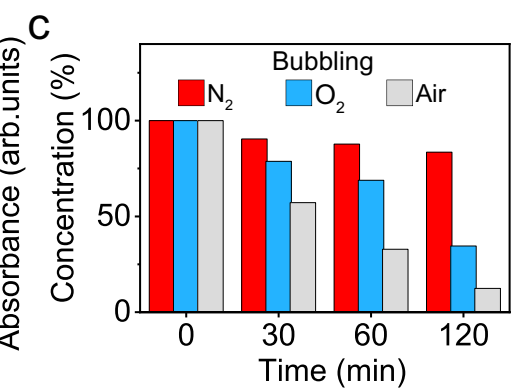

$f$

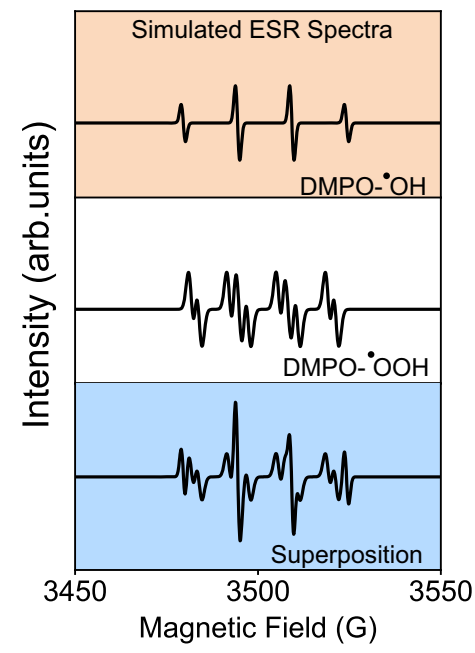

Fig. 3 Investigation on the contribution of radicals to degradation of MO. a Evolution of MO concentration in conditions of various radical scavengers. Ter-butanol, p-benzoquinone, $\mathrm{AgNO}_{3}$, and EDTA-2Na, are regarded as superoxide radical, hydroxide radical, electron, and proton scavengers, respectively. b Evaluation of the production of $\mathrm{OH}$ and $\mathrm{O}_{2}$ radicals and their reactivities by the fluorescence intensity of $\mathrm{THA}-\mathrm{OH}$ and the absorbance of formazan dye, respectively. c Investigation of the influence of dissolved gas in solution on the degradation of MO. d Schematic of the effect of introducing ter-butanol on the production of DMPO adducts. e Measured EPR spectra under various conditions, and every peak is labeled by corresponding patterns. $\mathbf{f}$ Simulated EPR spectra under the same condition by EasySpin. Error bars represent standard deviation based on three replicate data.

mainly due to the nonequilibrium status during the measurement. (Supplementary Fig. 11)

Mechanism of contact-electro-catalysis. Contact-electrocatalysis (CEC) was proposed as the mechanism for degrading $\mathrm{MO}$ in presence of FEP particles. The propagation of ultrasonic waves in solution provokes the formation of cavitation bubbles (CB). The collapse of cavitation bubbles is assumed to induce frequent contact electrification at the FEP-water interface, from which arises electron exchanges. The step-by-step illustrations are exhibited in Fig. 4a. A nucleus of $\mathrm{CB}$ is firstly formed during ultrasonication. Thereafter, the $\mathrm{CB}$, containing dissolved gas, grows from the nucleus until reaching a critical size. At this point, the collapse of the CB creates a high-pressure microjet that chases the previously adsorbed water molecules on the FEP surface. An electron is transferred from water to FEP upon contact, and the notation of FEP* is proposed to describe the charged state of FEP after separation from water. In the meantime, the enclosed $\mathrm{O}_{2}$ is released and grabs the electron from the charged surface of FEP* once they collide. FEP $^{*}$ retrieves its initial uncharged state after exchanging this electron to $\mathrm{O}_{2}$, and this cycle repeats itself as long as the emission of ultrasonic waves is sustained. The energy barriers for realizing these electron exchanges processes were assessed by Density Functional Theory (DFT). The specific calculation method is available in Supplementary Note 2. Considering $\mathrm{CE}$ is sensitive to external conditions, the high-pressure environment resulting from the collapse of the $\mathrm{CB}$ was also taken into consideration. In cases of water/FEP and $\mathrm{FEP}^{*} / \mathrm{O}_{2}$, calculations presented in Fig. $4 \mathrm{~b}$ reveal the energy barriers for electron transferring in these two scenarios decreased by $18.8 \%$ and $23.3 \%$ respectively. Therefore, the formation and collapse of CBs could not only induce contact-separation cycles but also facilitate electron transfers during CE. Figure $4 \mathrm{c}$ wraps up and illustrates the potential mechanism for degrading organic pollutants by CEC. On the one hand, single electron transfer (SET) between water and FEP during CE results in the formation of water radical cations. Thus generated water radical cations undergo a rapid proton transfer from water, forming hydronium cations and hydroxyl radicals ${ }^{36}$. On the other hand, electrons accumulated at $\mathrm{FEP}^{*}$ surface are captured by $\mathrm{O}_{2}$, forming $\cdot \mathrm{O}_{2}{ }^{-}$ radicals. Then, $\cdot \mathrm{O}_{2}{ }^{-}$are protonated into hydroperoxyl $\left(\mathrm{HO}_{2} \cdot\right)^{3},{ }^{37}$ leading to the formation of hydroxyl radicals by a chain reaction. Hydroxyl radicals generated at the end of both steps then react with organic pollutants in an aqueous solution.

Scalability and recyclability of the contact-electro-catalysts. No obvious diminution in the performances for the degradation of MO has been observed after recycling the FEP powder 5 times, as seen in Fig. 5a. Besides, this catalytic strategy can also be scaled up as exhibited in Fig. 5b. A slight decrease in kinetic constants for larger beakers is mainly due to the mismatch between ultrasonication power and the volume of solutions. Moreover, owing to the CE phenomenon's ubiquitous existence, a broad range of materials could be utilized to degrade organic pollutants based on CEC, and these materials are generally commercially available and inexpensive polymers. A compact 
a
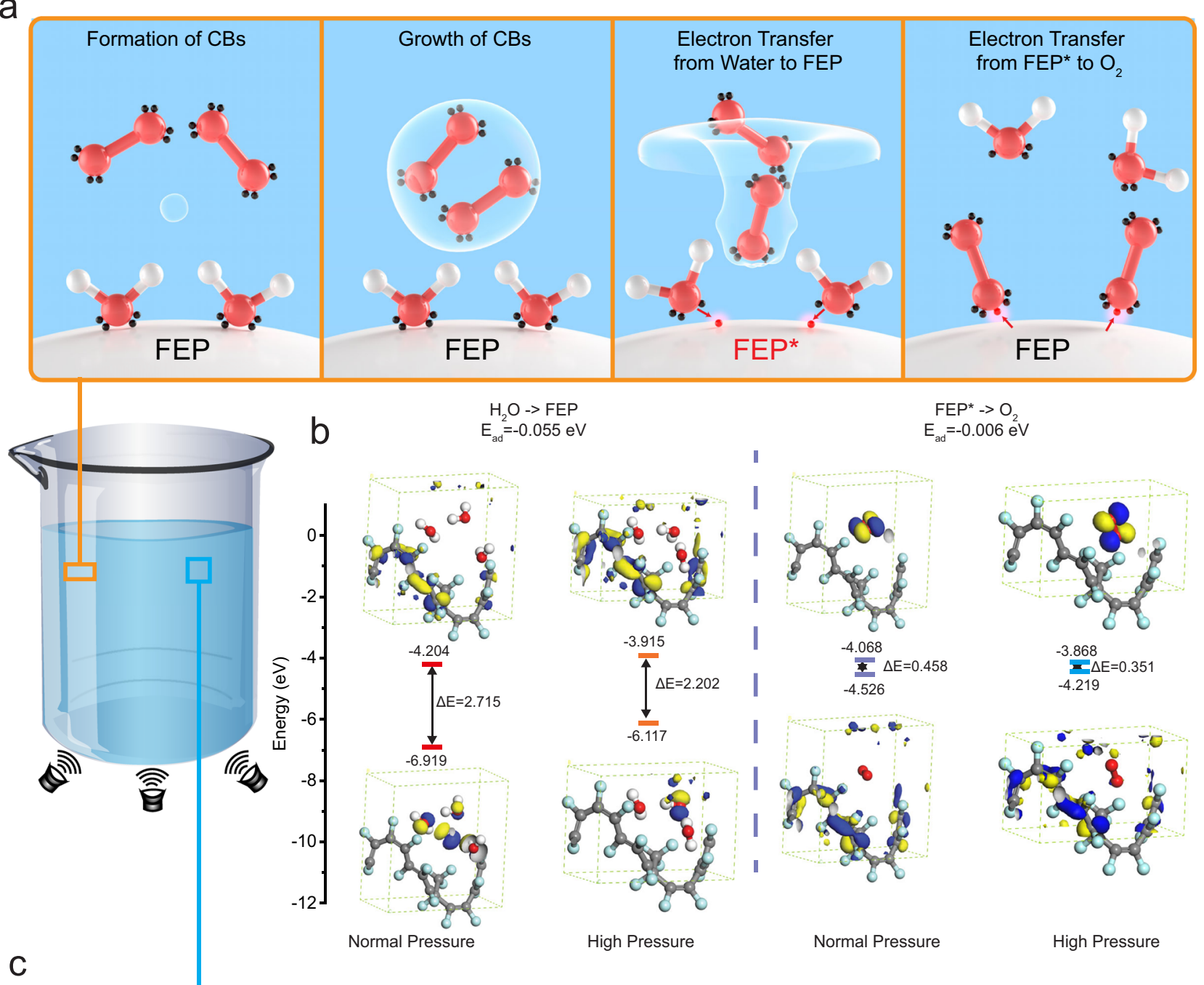

$b$

$$
\mathrm{E}_{\mathrm{ad}}=-0.055 \mathrm{eV}
$$

$E_{\mathrm{ad}}=-0.006 \mathrm{eV}$

C
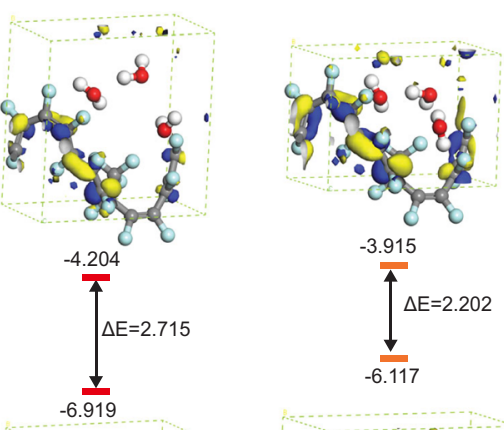

I
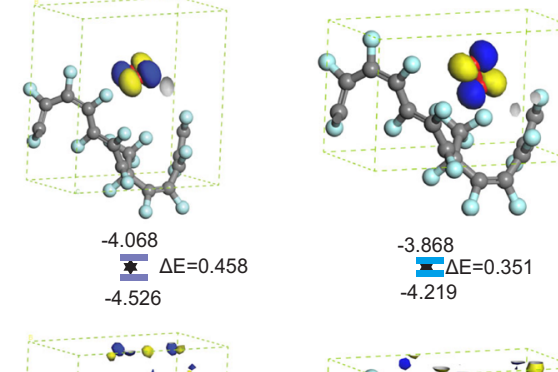

$-3.868$

들 $\Delta \mathrm{E}=0.351$

$-4.219$
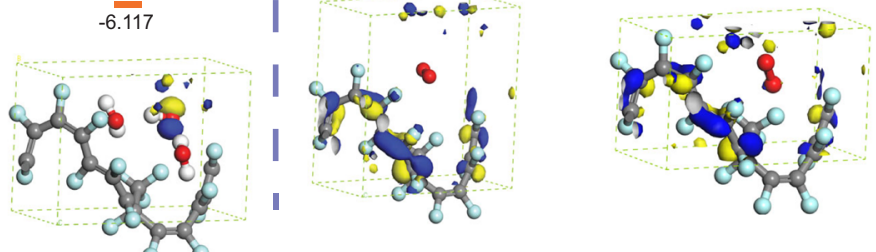

Normal Pressure

High Pressure

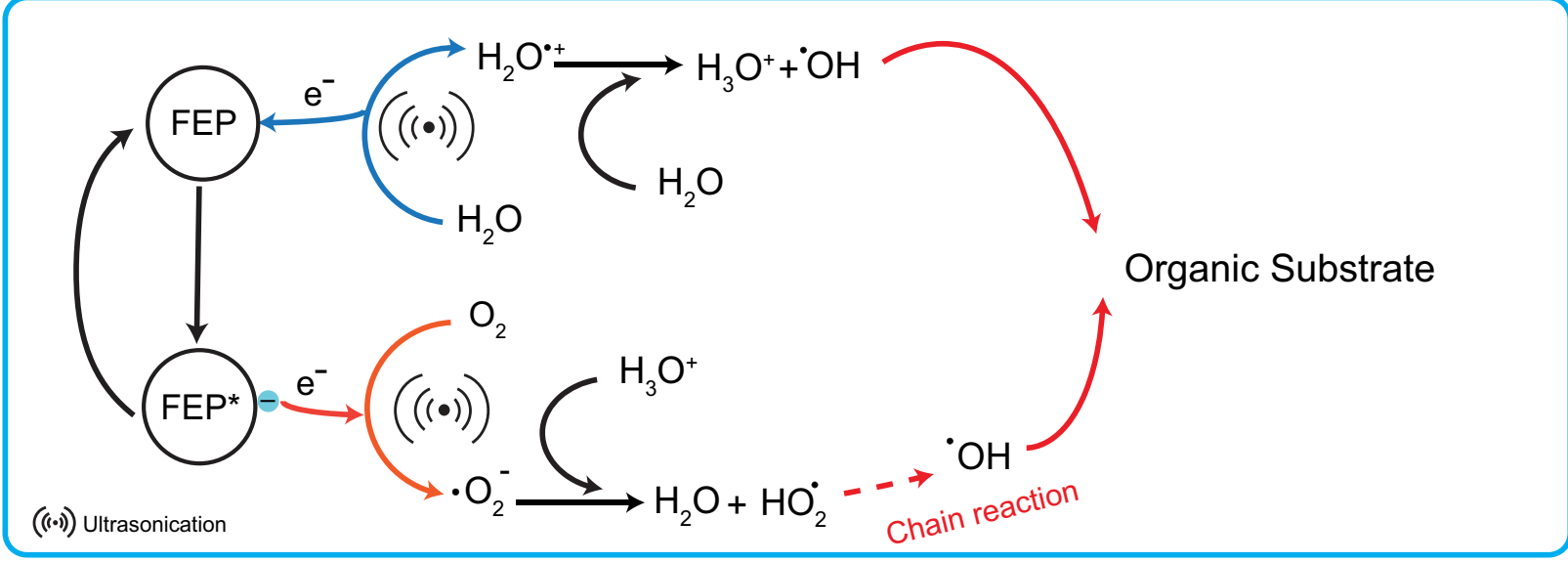

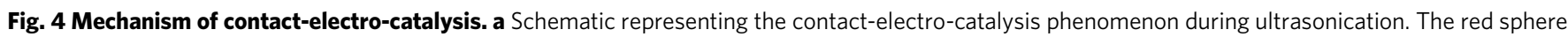
represents the oxygen atom, while the white for a hydrogen atom, and black for electrons. b DFT calculations of the values of LUMO and HOMO levels for Water-FEP and O2-FEP in various conditions as indicated by the legend. c Proposed mechanism for the degradation of MO by contact-electro-catalysis generated radicals.

architecture was devised for the cost-effective treatment of organic wastewater on the basis of the contact-electro-catalysis principle, as demonstrated in Fig. 5c. And we expect this attractive strategy to be a promising candidate for applications in chemical engineering, biological research, and fields that are closely related to ROS.

\section{Discussion}

A unique catalytic principle, contact-electro-catalysis (CEC), was first proposed and systematically investigated, which employs surface polarized electrons induced by contact electrification to accelerate chemical reactions. Ultrasonication-induced cavitation bubbles could not only generate the contact-separation cycles but 
a

\begin{tabular}{ccc}
$\begin{array}{c}\text { Cycle } \\
\text { Numbers }\end{array}$ & $\begin{array}{c}\text { Discoloration } \\
180 \mathrm{~min}\end{array}$ & $\begin{array}{c}\text { Kinectic Rate } \\
\left(\mathrm{min}^{-1}\right)\end{array}$ \\
\hline 1st cycle & 98.10 & 0.03956 \\
2nd cycle & 97.88 & 0.03359 \\
3rd cycle & 97.62 & 0.03848 \\
4th cycle & 98.88 & 0.04470 \\
5th cycle & 98.42 & 0.03582
\end{tabular}

b

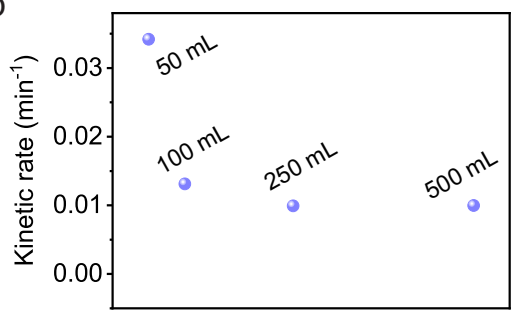

Increase of volume

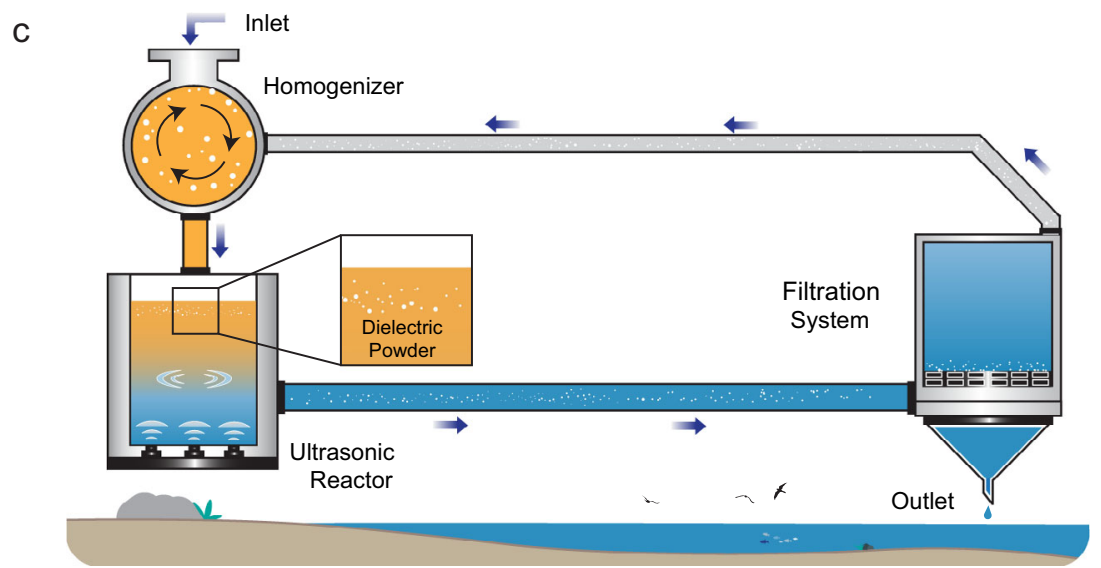

Fig. 5 Recyclability of contact-electro-catalysts. a Evolution of degradation from one to five cycles of reaction. $\mathbf{b}$ Investigations on the degradation rate for different volume MO solutions. c Expected large application of contact-electro-catalysis for the treatment of organic wastewater.

also facilitate electron transfer by decreasing energy barriers for various active species generation. Our results indicated a $50 \mathrm{~mL}$ 5-ppm MO aqueous solution was completely degraded after $3 \mathrm{~h}$ of ultrasonication in presence of $20 \mathrm{mg}$ of pristine FEP powder. And this catalytic efficiency could be further enhanced by introducing micro-nano structures on dielectric powder to increase the contact surface area or by chemical modifications as a mean to improve the surface charge density. As a ubiquitous phenomenon among various interfaces, contact electrification endows its derived contact-electro-catalysis with the power to greatly enrich the category of catalytic mechanisms and broaden the range of materials to be regarded as catalysts.

\section{Methods}

Chemical reagents. Methyl orange $\left[\mathrm{C}_{14} \mathrm{H}_{14} \mathrm{~N}_{3} \mathrm{NaO}_{3} \mathrm{~S}\right.$, Macklin, 98\%], acid orange$17\left[\mathrm{C}_{18} \mathrm{H}_{15} \mathrm{~N}_{2} \mathrm{NaO}_{4} \mathrm{~S}\right.$, Macklin], rhodamine $\mathrm{B}\left[\mathrm{C}_{28} \mathrm{H}_{31} \mathrm{ClN}_{2} \mathrm{O}_{3}\right.$, Macklin, 99\%], p-benzoquinone $\left[\mathrm{C}_{6} \mathrm{H}_{4} \mathrm{O}_{2}\right.$, Macklin $\left.99.5 \%\right]$, p-phthalic acid $\left[\mathrm{C}_{8} \mathrm{H}_{6} \mathrm{O}_{4}\right.$, Macklin, 99\%], sodium phosphate tribasic dodecahydrate $\left[\mathrm{Na}_{3} \mathrm{PO}_{4}\right.$, Aladdin, $\left.99.99 \%\right]$, ethylenediaminetetraacetic acid disodium salt dihydrate $\left[\mathrm{C}_{10} \mathrm{H}_{14} \mathrm{~N}_{2} \mathrm{Na}_{2} \mathrm{O}_{8} \cdot 2 \mathrm{H}_{2} \mathrm{O}\right.$, Aladdin, $99 \%$, tert-butanol $\left[\mathrm{C}_{4} \mathrm{H}_{10} \mathrm{O}\right.$, Sinopharm Chemical Reagent Co., Ltd, 98.0\%], silver nitrate $\left[\mathrm{AgNO}_{3}, 99.8 \%\right.$, Sinopharm Chemical Reagent Co., Ltd], 5,5dimethyl-1-pyrroline $\mathrm{N}$-oxide $\left[\mathrm{C}_{2} \mathrm{H}_{6} \mathrm{OS}\right.$, Dojindo], WST assay Kit $\mathrm{S} 311$ [Dojindo], superoxide dismutase from bovine [S5395-30KU, Sigma-Aldrich], potassium bomide [KBr, 99.997\%, Aladdin], fluorinated ethylene propylene (FEP) [Dupont], polytetrafluoroethylene (PTFE) [Dupont], polyvinylidene fluoride (PVDF)

[ $\left(\mathrm{C}_{2} \mathrm{H}_{2} \mathrm{~F}_{2}\right)$ n, SOLVAY], nylon-6,6 [ $\left(\mathrm{C}_{12} \mathrm{H}_{22} \mathrm{~N}_{2} \mathrm{O}_{2}\right) \mathrm{n}$, Dupont], nitrile butadiene rubber (NBR) [Kumho], and AlN from Macklin.

Sample preparation. A 5-ppm aqueous methyl orange solution was prepared by adding $5 \mathrm{mg}$ of $\mathrm{C}_{14} \mathrm{H}_{14} \mathrm{~N}_{3} \mathrm{NaO}_{3} \mathrm{~S}$ in $1 \mathrm{~L}$ of ultrapure water, followed by magnetic stirring for $1 \mathrm{~h}$.

About $20 \mathrm{mg}$ of polymer powder were added into a beaker containing $50 \mathrm{~mL}$ of the as-prepared methyl orange solution, and then magnetically stirred at $1000 \mathrm{rpm}$ for $48 \mathrm{~h}$. The solution containing MO and the powder was ultrasonicated $(40 \mathrm{kHz}$, $120 \mathrm{~W}$ ) using an ultrasonic bath (Yumeng, YM020S). Aliquots were sampled at 0 , $5,10,15,30,60,120,180 \mathrm{~min}$. The temperature in the ultrasonic bath was regulated.
The solution of terephthalic acid was prepared by adding $332.4 \mathrm{mg}$ of p-phthalic acid and $760 \mathrm{mg}$ of sodium phosphate tribasic dodecahydrate.

The WST-1 solution was prepared by diluting $1 \mathrm{ml}$ of WST-1 solution from a DOJINDO assay kit, into $19 \mathrm{~mL}$ of buffer solution and adding $30 \mathrm{~mL}$ of ultrapure water.

The powders after reactions were separated from the solution using a vacuum filtration system. The filtered powders were then dried in an oven at 40 degrees overnight before analysis.

FTIR samples were prepared by grinding $0.5 \mathrm{mg}$ of polymer powders with $100 \mathrm{mg}$ of $\mathrm{KBr}$ and then pressing them into a pellet.

Samples for EPR analysis were prepared by stirring $50 \mathrm{~mL}$ of ultrapure water and $20 \mathrm{mg}$ FEP powders for $48 \mathrm{~h}$ at $1000 \mathrm{rpm}$. About $0.5 \mathrm{~mL}$ of DMPO was transferred to the solution and stirred for $5 \mathrm{~min}$ at $500 \mathrm{RPM}$ prior to ultrasonication.

The MO solution for the scalability assessment was prepared as described above. The quantity of FEP powder was added proportionally to the volume of the solution treated. For instance, to treat a solution of $200 \mathrm{~mL}, 80 \mathrm{mg}$ of FEP powder were added.

Sample characterization. The UV-Vis absorbance of the aliquots was measured using a Shimadzu UV-3600 UV-Visible spectrometer on a range of 250-650 nm. The samples were placed into a Hellma Analytics QS High precision cell (Art. No. 104-10-40), with a light path of $10 \mathrm{~mm}$.

The emission spectra of THA-OH were measured on an Edinburgh Instruments FLS 980, using $\lambda_{\text {excitation }}=225 \mathrm{~nm}$ and $\lambda_{\text {emission }}=425 \mathrm{~nm}$.

The Scanning electron microscopy (SEM) images of the samples were obtained using an FEI Nova 450.

The Energy Dispersive X-Ray analysis (EDX) were conducted on FEI Nova 450 equipped with an AMETEK Octane Super appendix.

The X-ray photoelectron spectroscopy measurements have been conducted on a Thermo Fisher Scientific K-Alpha, in a vacuum of $1 \times 10^{-9} \mathrm{mBar}$, using an Alka ray source $(\mathrm{hv}=1486.6 \mathrm{eV})$, the working voltage is $15 \mathrm{kV}$ and the filament current is $10 \mathrm{~mA}$. The signal accumulation was performed for five to ten cycles. The pass energy is set at $30 \mathrm{eV}$.

The LC-MS analysis were conducted using a Thermo Scientific Q Exactive Orbitrap Quadrupole-Electrostatic Field Orbitrap High-Resolution Tandem Mass Spectrometer. The HESI ion source of the mass spectrometer was set at $-3.0 \mathrm{kV}$, in positive ion mode. The mass spectrometry scanner was set on the full scan range of $100-1000 \mathrm{~m} / \mathrm{z}$. The resolution of the instrument is 70000 FMHM. The column used was a Hypersil Gold C18 $(2.1 \times 100 \mathrm{~mm}, 1.9 \mu \mathrm{m})$, the column temperature is 
set at $40{ }^{\circ} \mathrm{C}$. The injection volume is $5 \mu \mathrm{L}$. Mobile phase A is composed of $0.1 \%$ formic acid aqueous solution, and mobile phase $\mathrm{B}$ is an acetonitrile solution.

The Raman spectroscopy analysis was conducted on a LabRam HR evolution (HORIBA, SAS France), using a range from 300 to $1400 \mathrm{~cm}^{-1}$.

FTIR analysis were conducted on a Bruker Vertex $80 \mathrm{v}$ on a range from 400 to $3000 \mathrm{~cm}^{-1}$

Electron paramagnetic resonance were recorded on a Bruker EMX plus-9.5/12/ $\mathrm{P} / \mathrm{L}$. The measurements were conducted in X-Band $(9.830243 \mathrm{GHz})$, with amplitude modulation of $1 \mathrm{G}$, microwave power of $2 \mathrm{~mW}$, and an amplitude modulation frequency of $100 \mathrm{kHz}$ and conversion time of $60 \mathrm{~ms}$, and a time constant at $40.96 \mathrm{~ms}$. The assignment of the components of the spectra was based on literature and simulation using Easyspin. The code used for the simulation of the spectra is presented in Supplementary Note 1.

\section{Data availability}

The data supporting the findings of this study are reported in the main text or the Supplementary Information. Raw data can be obtained from the corresponding authors upon reasonable request.

Received: 15 July 2021; Accepted: 3 December 2021; Published online: 10 January 2022

\section{References}

1. Gomollón-Bel, F. Ten chemical innovations that will change our world: IUPAC identifies emerging technologies in Chemistry with potential to make our planet more sustainable. Chem. Int. 41, 12-17 (2019).

2. Xing, T. et al. Gas protection of two-dimensional nanomaterials from highenergy impacts. Sci. Rep. 6, 35532 (2016).

3. Lin, I. J. \& Nadiv, S. Review of the phase transformation and synthesis of inorganic solids obtained by mechanical treatment (mechanochemical reactions). Mater. Sci. Eng. 39, 193-209 (1979).

4. Boldyrev, V. V. \& Tkáčová, K. Mechanochemistry of solids: past, present, and prospects. J. Mater. Synth. Process. 8, 121-132 (2000)

5. Akbulatov, S. et al. Experimentally realized mechanochemistry distinct from force-accelerated scission of loaded bonds. Science 357, 299-303 (2017).

6. Hermann, G. N., Becker, P. \& Bolm, C. Mechanochemical iridium(III)catalyzed $\mathrm{C}-\mathrm{H}$ bond amidation of benzamides with sulfonyl azides under solvent-free conditions in a ball mill. Angew. Chem. Int. Ed. 55, 3781-3784 (2016).

7. McKissic, K. S., Caruso, J. T., Blair, R. G. \& Mack, J. Comparison of shaking versus baking: further understanding the energetics of a mechanochemical reaction. Green. Chem. 16, 1628-1632 (2014).

8. Xia, H. \& Wang, Z. Piezoelectricity drives organic synthesis. Science 366, 1451-1452 (2019).

9. Wang, Y. et al. Piezo-catalysis for nondestructive tooth whitening. Nat. Commun. 11, 1328 (2020).

10. Starr, M. B. \& Wang, X. Fundamental analysis of piezocatalysis process on the surfaces of strained piezoelectric materials. Sci. Rep. 3, 2160 (2013).

11. Zhao, J. et al. Strong tribo-catalysis of zinc oxide nanorods via triboelectrically-harvesting friction energy. Ceram. Int. 46, 25293-25298 (2020).

12. Li, P. et al. Strong tribocatalytic dye decomposition through utilizing triboelectric energy of barium strontium titanate nanoparticles. Nano Energy 63, 103832 (2019).

13. Yang, B. et al. Enhanced tribocatalytic degradation using piezoelectric $\mathrm{CdS}$ nanowires for efficient water remediation. J. Mater. Chem. C. 8, 14845-14854 (2020).

14. Liu, X. et al. Low frequency hydromechanics-driven generation of superoxide radicals via optimized piezotronic effect for water disinfection. Nano Energy 88, 106290 (2021).

15. Pan, M., Liu, S., Pan, B. \& Chew, J. W. Directionally tailoring the macroscopic polarization of piezocatalysis for hollow zinc sulfide on dual-doped graphene. Nano Energy 88, 106312 (2021).

16. Feng, Y. et al. Engineering spherical lead zirconate titanate to explore the essence of piezo-catalysis. Nano Energy 40, 481-486 (2017).

17. Kim, J. H. et al. A radical approach for the selective $\mathrm{C}-\mathrm{H}$ borylation of azines. Nature 595, 1-9 (2021).

18. Fu, N., Sauer, G. S., Saha, A., Loo, A. \& Lin, S. Metal-catalyzed electrochemical diazidation of alkenes. Science 357, 575-579 (2017).

19. Zhou, H. et al. Isolated boron in zeolite for oxidative dehydrogenation of propane. Science 372, 76-80 (2021).

20. Liang, J., Liang, Z., Zou, R. \& Zhao, Y. Heterogeneous catalysis in zeolites, mesoporous silica, and metal-organic frameworks. Adv. Mater. 29, 201701139 (2017).
21. Takata, T. et al. Photocatalytic water splitting with a quantum efficiency of almost unity. Nature 581, 411-414 (2020).

22. Ghosh, I. et al. Organic semiconductor photocatalyst can bifunctionalize arenes and heteroarenes. Science 365, 360-366 (2019).

23. Tu, S. et al. Piezocatalysis and piezo-photocatalysis: catalysts classification and modification strategy, reaction mechanism, and practical application. Adv. Funct. Mater. 30, 2005158 (2020).

24. Kubota, K., Pang, Y., Miura, A. \& Ito, H. Redox reactions of small organic molecules using ball milling and piezoelectric materials. Science 366, 1500-1504 (2019)

25. Zhan, F. et al. Electron transfer as a liquid droplet contacting a polymer surface. ACS Nano 14, 17565-17573 (2020).

26. Nie, J. et al. Probing contact-electrification-induced electron and ion transfers at a liquid-solid interface. Adv. Mater. 32, 1905696 (2020).

27. $\mathrm{Xu}, \mathrm{W}$. et al. A droplet-based electricity generator with high instantaneous power density. Nature 578, 392-396 (2020).

28. $\mathrm{Li}$, S. et al. Contributions of different functional groups to contact electrification of polymers. Adv. Mater. 32, 2001307 (2020).

29. Lin, S., Xu, L., Chi Wang, A. \& Wang, Z. L. Quantifying electron-transfer in liquid-solid contact electrification and the formation of electric double-layer. Nat. Commun. 11, 399 (2020).

30. Yasui, K. Acoustic Cavitation and Bubble Dynamics (Springer, 2017).

31. Liu, C. \& Bard, A. J. Electrostatic electrochemistry at insulators. Nat. Mater. 7, 505-509 (2008).

32. Zhang, J. et al. Electrochemistry on tribocharged polymers is governed by the stability of surface charges rather than charging magnitude. J. Am. Chem. Soc. 141, 5863-5870 (2019).

33. Chamberlayne, C. F. \& Zare, R. N. What role does the electric double layer play in redox reactions at planar electrostatically charged insulating surfaces? Top. Catal. 64, 1-6 (2021).

34. Sun, L. L., Lin, S. Q., Tang, W., Chen, X. \& Wang, Z. L. Effect of redox atmosphere on contact electrification of polymers. ACS Nano 14, 17354-17364 (2020).

35. Finkelstein, E., Rosen, G. M. \& Rauckman, E. J. Spin trapping of superoxide and hydroxyl radical: practical aspects. Arch. Biochem. Biophys. 200, 1-16 (1980).

36. Ma, J., Wang, F. \& Mostafavi, M. Ultrafast chemistry of water radical cation, $\mathrm{H}(2) \mathrm{O}(*+)$, in aqueous solutions. Molecules 23, 244 (2018).

37. Hayyan, M., Hashim, M. A. \& AlNashef, I. M. Superoxide ion: generation and chemical implications. Chem. Rev. 116, 3029-3085 (2016).

\section{Acknowledgements}

We would like to thank Dr. Shiquan Lin for his technical assistance. We appreciate Jianzhe Luo, Yuan Liu, Jie An, Chengyu Li, Sheng Shu, Di Liu, Xin Zhao, and Pengfei Chen for their assistance with collecting and organizing data. National Key R\&D Project from Minister of Science and Technology (Grant No. 2016YFA0202704, Z.L.W.), National Natural Science Foundation of China (Grant Nos. 51432005 and 5151101243, Z.L.W.), Beijing Municipal Science and Technology Commission (Grant Nos. Z181100003818016, Z171100000317001, Z171100002017017, and Y3993113DF, W.T.), Youth Innovation Promotion Association (W.T.), CAS-TWAS President's Fellowship (A.B.)

\section{Author contributions}

W.T. and Z.L.W. conceived the idea and supervised the experiment. Z.W., A.B., S.L., Y.F., L.Z., W.T., and Z.L.W. prepared the manuscript. Z.W., A.B., and Y.F. developed the experimental setups. Z.W. and A.B. performed data measurements. All the authors discussed the results and commented on the manuscript.

\section{Competing interests}

Z.L.W., W.T., Z.W., and A.B. are inventors of the Patent (202110606121.0) that was filed on 31 May 2021 by the Beijing Institute of Nanoenergy and Nanosystems, Chinese Academy of Sciences. The remaining authors declare no competing interests.

\section{Additional information}

Supplementary information The online version contains supplementary material available at https://doi.org/10.1038/s41467-021-27789-1.

Correspondence and requests for materials should be addressed to Wei Tang or Zhong Lin Wang.

Peer review information Nature Communications thanks Koji Kubota and the other, anonymous, reviewers for their contribution to the peer review of this work.

Reprints and permission information is available at http://www.nature.com/reprints

Publisher's note Springer Nature remains neutral with regard to jurisdictional claims in published maps and institutional affiliations. 
(c) (i) Open Access This article is licensed under a Creative Commons Attribution 4.0 International License, which permits use, sharing, adaptation, distribution and reproduction in any medium or format, as long as you give appropriate credit to the original author(s) and the source, provide a link to the Creative Commons license, and indicate if changes were made. The images or other third party material in this article are included in the article's Creative Commons license, unless indicated otherwise in a credit line to the material. If material is not included in the article's Creative Commons license and your intended use is not permitted by statutory regulation or exceeds the permitted use, you will need to obtain permission directly from the copyright holder. To view a copy of this license, visit http://creativecommons.org/ licenses/by/4.0/.

(C) The Author(s) 2022 